\title{
Survival and quality of life for patients with COPD or asthma admitted to intensive care in a UK multicentre cohort: the COPD and Asthma Outcome Study (CAOS)
}

\author{
M J Wildman, ${ }^{1}$ C F B Sanderson, ${ }^{2}$ J Groves, ${ }^{3}$ B C Reeves, ${ }^{2}$ J G Ayres, ${ }^{4}$ D Harrison, ${ }^{5}$ \\ D Young, ${ }^{6} \mathrm{~K}$ Rowan ${ }^{5}$
}

- Tables 4 and 5 are published online only at http://thorax.bmj. com/content/vol64/issue2

${ }^{1}$ Sheffield Thoracic Institute, Northern General Hospital, Sheffield, UK; ${ }^{2}$ Health Services Research Unit, London School of Hygiene and Tropical Medicine, London, UK; ${ }^{3}$ Heartlands Hospital, Birmingham, UK ; ${ }^{4}$ Department of Environmental and Occupational Medicine, University of Aberdeen, UK; ${ }^{5}$ Intensive Care National Audit and Research Centre, London UK; ${ }^{6}$ Nuffield Department of Anaesthetics, Oxford, UK

Correspondence to: Dr M J Wildman, Sheffield Thoracic Institute, Northern General Hospital, Herries Road Sheffield S5 7AU, UK; martin. wildman@sth.nhs.uk

Received 30 September 2007 Accepted 23 September 2008 Published Online First 13 October 2008

\section{ABSTRACT}

Background: Non-invasive ventilation is first-line treatment for patients with acutely decompensated chronic obstructive pulmonary disease (COPD), but endotracheal intubation, involving admission to an intensive care unit, may sometimes be required. Decisions to admit to an intensive care unit are commonly based on predicted survival and quality of life, but the information base for these decisions is limited and there is some evidence that clinicians tend to be pessimistic. This study examined the outcomes in patients with COPD admitted to the intensive care unit for decompensated type II respiratory failure.

Methods: A prospective cohort study was carried out in 92 intensive care units and 3 respiratory high dependency units in the UK. Patients aged 45 years and older with breathlessness, respiratory failure or change in mental status due to an exacerbation of COPD, asthma or a combination of the two were recruited. Outcomes included survival and quality of life at 180 days.

Results: Of the 832 patients recruited, 517 (62\%) survived to 180 days. Of the survivors, 421 (81\%) responded to a questionnaire. Of the respondents, $73 \%$ considered their quality of life to be the same as or better than it had been in the stable period before they were admitted, and 96\% would choose similar treatment again. Function during the stable pre-admission period was a reasonable indicator of function reported by those who survived 180 days.

Conclusions: Most patients with COPD who survive to 180 days after treatment in an intensive care unit have a heavy burden of symptoms, but almost all of themincluding those who have been intubated-would want similar intensive care again under similar circumstances.

An acute exacerbation of chronic obstructive pulmonary disease (COPD) is a common reason for hospital admission, and although non-invasive ventilation is the recommended first-line treatment for decompensated type II respiratory failure, on occasions endotracheal intubation may be required. ${ }^{1}$ Decision making about intubation should involve some understanding of the likely probability of survival, and also of the quality of life that survivors can expect. This information will be most useful if it comes from systematic studies of survivors, who provide both assessments of their own quality of life and their views about the acceptability of their treatment. There are some data from the USA ${ }^{2}$ but very few from the UK, which has historically had fewer intensive care unit (ICU) beds per unit population and may have different admission policies and practices. A simulation study carried out in one Critical Care Network in the UK suggested that, for patients with COPD with a given set of characteristics and preferences and subject to identical resource constraints, there was variation in decision making about whether intubation was appropriate, partly because of widely differing estimates of the probability of survival. ${ }^{3}$ Moreover, an analysis of actual decision making in the UK suggested that clinicians tended to be pessimistic about survival. ${ }^{4}$ In order to provide both clinicians and patients with better information about outcomes for COPD after critical care, this study reports the survival, quality of life and future treatment preferences of patients in the COPD and Asthma Outcome Study (CAOS) study.

\section{METHODS}

\section{Intensive care units and subjects}

All ICUs participating in the UK Case Mix Programme $(\mathrm{CMP})^{5}$ and three respiratory high dependency units (HDUs) in hospitals with ICUs were invited to take part in the study. Patients were eligible if they were admitted to a participating unit with breathlessness, respiratory failure or change in mental status due to an exacerbation of obstructive lung disease. They were recruited on admission to the unit. Clinicians were asked to classify the patient as having either COPD, asthma or a mixture of the two, because the precise classification of obstructive airways disease can be difficult at the time decisions about admission to ICU have to be made, but even an imprecise diagnosis may have prognostic value. Clinicians found this classification easy to use in the clinical setting.

Patients under the age of 45 years were excluded as it was expected that they would be admitted irrespective of prognosis. Patients were also excluded if they had had surgery within the past 10 days or had been transferred from another hospital. Data were collected for admissions between March 2002 and September 2003 with follow-up for 180 days after ICU admission.

\section{Collection of data}

Data were sought about each patient's function during the period of stability 2 weeks before 
admission using a 4-point scale, ${ }^{6}$ and standard questions were asked about activities of daily living. ${ }^{7}$ Clinicians were asked to use the EuroOol visual analogue score $(\mathrm{VAS})^{8}$ to predict the patients' self-rated quality of life at 180 days if they were to survive.

When a patient was discharged from a unit or died while in it without having been intubated, clinicians were asked whether intubation had been ruled out as a treatment option if medical treatment and non-invasive ventilation were to fail or had failed. This information was used to divide the patients into three "intubation status" groups: (1) intubation not needed; (2) intubated; and (3) not to be intubated. This last group included those for whom clinicians felt intubation to be futile and patients who declind treatment escalation.

Actual survival to 180 days was determined initially from the general practitioner and confirmed by the Office of National Statistics. A follow-up questionnaire was sent to patients who survived 180 days which included the 4-point functional score used on admission, the Euroqol profile and VAS, the AO-20, ${ }^{9}$ and how their current state of health compared with their health during the period of stability before hospitalisation. In addition, to understand how patients felt about their experience of the ICU and intubation, they were asked: "Under the same circumstances, would you be willing to undergo similar intensive care treatment again?"

Data for the participating ICUs and others were also obtained from the CMP database.

\section{Analysis of data}

Data from the study were compared with extracts from the CMP database to assess how representative of the UK were the ICU/HDUs and patients recruited. Data from the CMP were also used to indicate the variation between units in admission policies.

Survival rates and quality of life on follow-up were examined overall and for the three "intubation status" groups. Clinicians' predictions of quality of life among 180-day survivors were compared with the survivors' assessments, and functional status in the 2 weeks before admission was compared with survivors' reports.

Table 1 Intensive care units in the Case Mix Programme (CMP) participating and not participating in the COPD and Asthma Outcome Study (CMP data)

\begin{tabular}{lll}
\hline & Participating & Not participating \\
\hline $\begin{array}{l}\text { Total number of units } \\
\text { Type of unit }\end{array}$ & $89(100 \%)$ & $76(100 \%)$ \\
ICU & $41(46.1 \%)$ & $27(35.5 \%)$ \\
ICU/HDU & $42(47.2 \%)$ & $39(51.3 \%)$ \\
$\quad$ Other & $6(6.7 \%)$ & $10(13.2 \%)$ \\
Number of beds & & \\
$3-5$ & $21(23.6 \%)$ & $15(19.7 \%)$ \\
$6-9$ & $43(48.3 \%)$ & $41(53.9 \%)$ \\
$10+$ & $25(28.1 \%)$ & $20(26.3 \%)$ \\
In university or affiliated hospitals & $35(39.7 \%)$ & $32(42.1 \%)$ \\
Admissions per unit per year & 470 & 454 \\
Total admissions during study period $\left(\mathrm{n}_{1}\right)$ & 39309 & 46258 \\
Admissions potentially eligible for study & & \\
$\quad \mathrm{n}_{2}\left(\%\right.$ of $\left.\mathrm{n}_{1}\right)$ & $1543(3.9 \%)$ & $1501(3.2 \%)$ \\
Mean (SD) CAPS & $33.6(12.0)$ & $35.1(12.5)$ \\
ICU survival (\% of $\left.\mathrm{n}_{2}\right)$ & $1223(79.3 \%)$ & $1138(75.8 \%)$ \\
\hline
\end{tabular}

CAPS, COPD acute physiology score; HDU, high dependency unit; ICU, intensive care unit.
Analyses were carried out using Stata Version 9 (Stata Corp, College Station, Texas, USA).

\section{RESULTS}

\section{Units and patients recruited}

Of the 239 ICUs in the UK in January 2002, the 177 contributing to the CMP database at the time were invited to take part. Three were ineligible because they were specialist units that never admitted patients with COPD, 73 refused, 3 did not complete the ethics process and 6 recruited no patients. Thus, 92 ICUs actively participated in the study, $38.5 \%$ of the units in the UK and $52.0 \%$ of those invited. Three respiratory ICUs also participated.

For 89 of the 92 ICUs, data were available from the CMP database to allow comparison with non-participating units. The units actively participating were similar to others in terms of type, size and affiliation, and the mean percentages of admissions potentially eligible for the study (table 1 ). The CMP database also allowed comparisons of the patients recruited with those eligible but not recruited in the participating units (table 2). A total of 832 patients were recruited, 724 to ICUs and 108 to respiratory HDUs. At the time of these analyses there were data in the CMP dataset for 648 of the 724 (94.5\%). These 648 were compared with the 996 patients in the dataset admitted to participating ICUs and satisfying the inclusion criteria but not recruited. There were no differences in terms of age (Z-test, $p=0.49)$, COPD acute physiology $\operatorname{score}^{10}$ (Z-test, $\left.\mathrm{p}=0.96\right)$ or ICU survival $\left(\chi^{2} 0.77, \mathrm{p}=0.38\right)$.

The CMP database had data on 8717 patients who met the CAOS study inclusion criteria and were admitted during the period 2000-6 to the ICUs in the CAOS study. The participating centres are plotted from left to right by increasing mean age in fig 1 and by increasing percentage of admitted patients in the most limited classification of baseline respiratory impairment in APACHE II in fig 2. These diagrams suggest between-unit variation in the age and fitness of patients admitted. This may be partly due to variation between catchment populations, but it also suggests that some units are more selective than others about which patients with COPD/asthma they admit.

\section{Ventilation and length of stay}

Of the 832 patients recruited, $81.3 \%$ were admitted only to the ICU. $54.1 \%$ were intubated before or during ICU admission, $36.5 \%$ were not intubated but had non-invasive ventilation, and $8.1 \%$ had medical treatment only. For 201 (53\%) of the nonintubated patients, medical treatment and non-invasive ventilation were sufficient to manage the exacerbation ("intubation not needed"), but in the other $47 \%$ the patients were designated "not to be intubated".

Baseline differences between intubation status groups are given in table 3 . In the intubated and intubation not needed

Table 2 Potentially eligible patients in participating units recruited and not recruited (data from Case Mix Programme (CMP))

\begin{tabular}{lll}
\hline & $\begin{array}{l}\text { Recruited } \\
(\mathbf{n}=\mathbf{6 4 8})\end{array}$ & $\begin{array}{l}\text { Not recruited } \\
(\mathbf{n}=\mathbf{9 9 6})\end{array}$ \\
\hline Mean (SD) age (years) & $66.2(9.9)$ & $66.8(10.2)$ \\
Mean (SD) CAPS & $33.9(12.2)$ & $33.9(12.1)$ \\
Men (\%) & 48.8 & 49.2 \\
Admission out of hours (18.00-08.00 h) (\%) & 49.7 & 52.8 \\
Admission at weekend (\%) & 26.7 & 26.7 \\
ICU survival (\%) & 80.6 & 78.2 \\
\hline
\end{tabular}

CAPS, COPD acute physiology score; ICU, intensive care unit. 


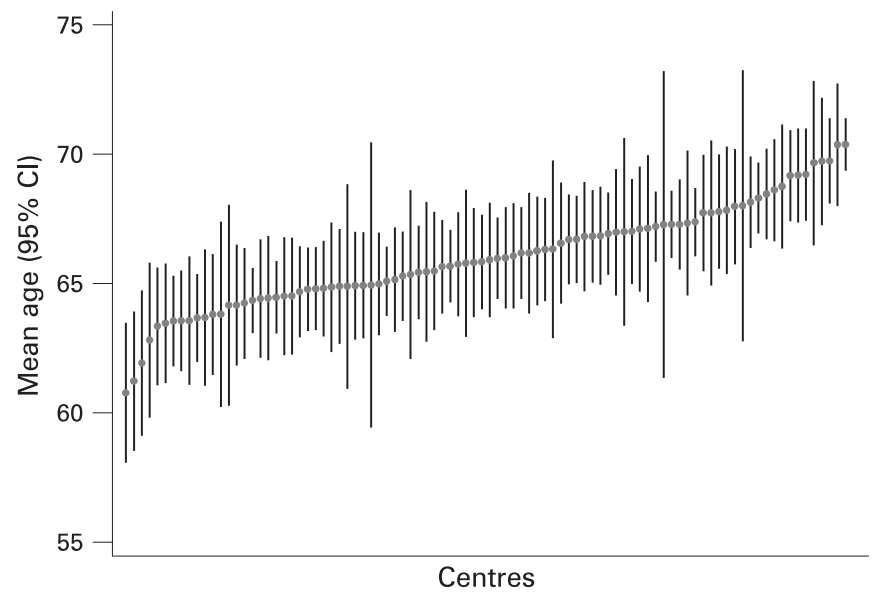

Figure 1 Mean age (with 95\% confidence limits) of patients with chronic obstructive pulmonary disease admitted to the intensive care units involved in the COPD and Asthma Outcome Study during the period 2000-6 $(\mathrm{n}=8717)$.

groups the patients have been subdivided into those with COPD or a mixture of COPD and asthma (labelled "COPD \pm ") and those diagnosed on admission as "pure asthma". (In the "not to be intubated" group there were only two patients with pure asthma, and only one who responded to the 180-day questionnaire, so this group was not subdivided.) In table 3, as elsewhere, the numbers in the intubation not needed/pure asthma subgroup were small and so the parameter estimates will be very imprecise. Of the patients designated "not to be intubated", $57 \%$ had been admitted directly to the ICU. They tended to be older than the others (mean age 70.3 vs 66.1 years, $t$ test, $\mathrm{p}<0.001)$ and had slightly lower mean COPD acute physiology scores (26.4 vs $28.4, t$ test, $p=0.03$ ). They had much lower functional scores (housebound or worse $68.0 \%$ vs $30.0 \%$, $\chi^{2}$ test, $\mathrm{p}<0.001$ ), and $75.1 \%$ were either housebound or on long-term oxygen or both compared with $32.7 \%$ in the intubated group.

The intubated patients had longer stays than the others, both in the COPD \pm and the pure asthma subgroups.

\section{Survival}

Survival rates at discharge from the ICU and from hospital and at 180 days after ICU admission are given in table 3. Overall, $517(62.1 \%)$ survived to 180 days. Survival rates were highest in patients who did not need intubation and lowest in those designated "not to be intubated", but the differences in survival between the intubated COPD \pm and the "not to be intubated" groups were quite small, with overlapping confidence intervals. The intraclass correlation for actual survival rates between participating units was 0.017 ( $95 \%$ CI 0.000 to 0.057 ), so no adjustment was made to the confidence intervals for unit-level effects.

\section{Quality of life at $\mathbf{1 8 0}$ days}

Of the 517 subjects who survived to 180 days, 420 (81.4\%) returned the follow-up questionnaire. The median (IOR) time from admission to the ICU to return of the questionnaire was 231 days (200-399). There was no evidence for a difference in the percentage of survivors returning the questionnaire between the five groups examined (see table 4 available online). Overall, $73 \%$ of respondents stated that their current state of health was the same as or better than before hospitalisation; the figures for

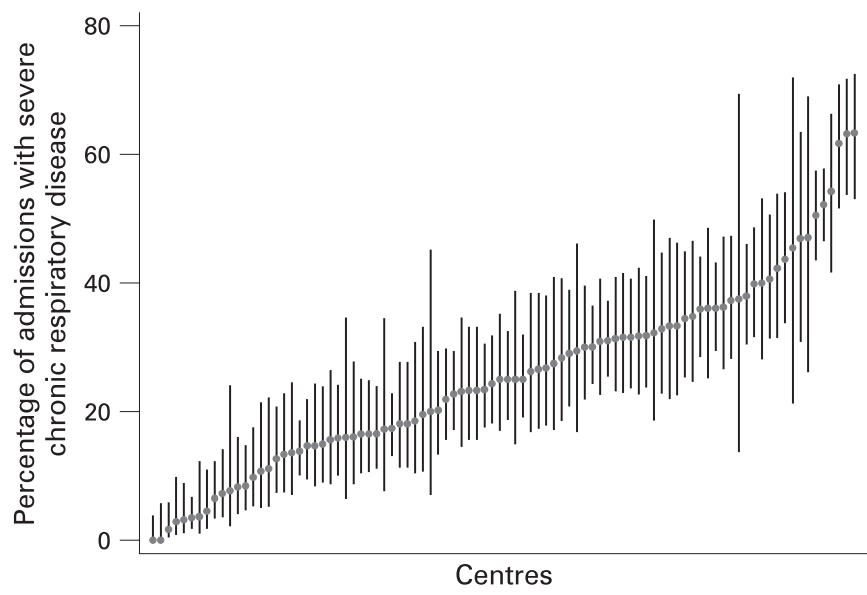

Figure 2 Percentage (with 95\% confidence limits) of patients with chronic obstructive pulmonary disease and severe respiratory disease in the period of stability before admission to the intensive care units participating in the COPD and Asthma Outcome Study during the period 2000-6 $(n=8717)$. Severe chronic respiratory disease is defined as "permanent shortness of breath with light activity due to pulmonary disease. Functionally, this patient is unable to work and has shortness of breath performing most normal activities of daily living, for example, walking 20 metres on level ground, walking slowly in the house, climbing one flight of stairs, dressing or standing".

the 184 prompt responders (<220 days after admission), 179 delayed responders (221-365 days) and 57 very delayed responders were $71 \%, 75 \%$ and $70 \%$, respectively, giving no indication that non-responders might have responded markedly differently $\left(p=0.81, \chi^{2}\right.$ test $) .{ }^{11}$

Table 4 (online) also gives results from the EuroQol schedule. For example, $62 \%$ of the respondents experienced moderate or extreme discomfort and 56\% had some anxiety/depression, $7 \%$ rating this as extreme. Generally, the quality of life in the notto-be-intubated group was poorer than for the rest. On the Euroaol VAS $(0=$ "worst possible health state", $100=$ "the best imaginable health state") the overall mean (SD) rating was 54.9 (19.5); $75 \%$ rated their health state as at least 40 and $50 \%$ rated it as at least 50 .

The AQ-20 questionnaire gives more detail about the symptoms associated with airways disease and table 5 (available online) summarises the data from the 420 respondents. The question on doing things at work was only answered by 59 people so the results are unreliable and are only included for completeness. The $p$ values are for $\chi^{2}$ tests of the hypothesis of no difference in the percentages between intubation status groups. $42.7 \%$ of the respondents reported feeling breathless when trying to get to sleep. Only $16 \%$ of those who considered the question applicable could get upstairs without breathlessness, $74 \%$ had difficulty getting around the house because of their chest trouble, and $83 \%$ felt that the fullness of their lives was limited by their chest troubles. For the AQ-20 score, which has a range of $0-20$, the mean (SD) and median (IOR) scores were 11.2 (4.7) and 12 (8-15), respectively.

\section{Willingness to undergo similar treatment in the future}

In spite of this burden of symptoms and disabilities, $96 \%$ of the 415 who answered the question about whether they would be willing to undergo similar treatment again under the same circumstances said that they would. In the 212 intubated patients the percentage was the same. Even under the worstcase assumption that all non-responders would not want ICU 
Table 3 Recruitment, baseline values, ventilation, survival and questionnaire response

\begin{tabular}{|c|c|c|c|c|c|c|c|}
\hline & \multicolumn{2}{|c|}{ Intubation not needed } & \multicolumn{2}{|l|}{ Intubated } & \multirow{2}{*}{$\begin{array}{l}\text { Not to be } \\
\text { intubated } \\
(\mathrm{n}=181)\end{array}$} & \multirow[b]{2}{*}{$\begin{array}{l}\text { Overall } \\
(n=832)\end{array}$} & \multirow[b]{2}{*}{ p Value $\S$} \\
\hline & $\begin{array}{l}\text { COPD } \pm \\
(n=179)\end{array}$ & $\begin{array}{l}\text { "Pure" asthma } \\
(\mathrm{n}=22)\end{array}$ & $\begin{array}{l}\text { COPD } \pm \\
\text { (n = 394) }\end{array}$ & $\begin{array}{l}\text { "Pure" asthma } \\
(\mathrm{n}=56)\end{array}$ & & & \\
\hline $\begin{array}{l}\text { Admitted directly to } \\
\text { ICU* (\%) }\end{array}$ & 60.3 & 77.3 & 99.5 & 100.0 & 56.9 & 81.3 & \\
\hline \multicolumn{8}{|l|}{ On admission } \\
\hline Mean (SD) age (years) & $67.7(9.2)$ & $59.1(10.6)$ & $66.8(8.9)$ & $57.9(9.7)$ & $70.3(9.4)$ & $67(9.7)$ & \\
\hline Mean (SD) CAPS & $23.9(9.9)$ & $22.4(10.6)$ & $31(10.8)$ & $26.4(9.8)$ & $26.4(10.2)$ & $27.9(10.8)$ & \\
\hline On long-term oxygen† $(\%)$ & 12.9 & 9.1 & 10.4 & 5.4 & 38.1 & 16.6 & \\
\hline \multicolumn{8}{|l|}{ Ventilation } \\
\hline Non-invasive only (\%) & 74.7 & 13.6 & 0.0 & 0.0 & 91.7 & 36.6 & $<0.001$ \\
\hline \multicolumn{8}{|l|}{$\begin{array}{l}\text { Median (IQR) length of stay } \\
\text { (days) }\end{array}$} \\
\hline Surviving hospital (\%) & $93.9(89.3$ to 96.9$)$ & 95.5 (77.2 to 99.9$)$ & $61.2(56.2$ to 66$)$ & 85.7 (73.8 to 93.6$)$ & 59.7 (52.1 to 66.9$)$ & $70.4(67.2$ to 73.5$)$ & $<0.001$ \\
\hline Surviving 180 days (\%) & 81.6 (75.1 to 87$)$ & 95.5 (77.2 to 99.9$)$ & $54.6(49.5$ to 59.6$)$ & 85.7 (73.8 to 93.6$)$ & 48.1 (40.6 to 55.6 ) & 62.1 (58.7 to 65.4 ) & $<0.001$ \\
\hline
\end{tabular}

CAPS, COPD acute physiology score; HDU, high dependency unit; ICU, intensive care unit; IQR, interquartile range; MAC, mid-arm circumference.

${ }^{*}$ Not admitted to a respiratory support unit at any stage.

†0xygen concentrator at home before admission.

\$With exact $95 \%$ confidence intervals.

$\xi \chi^{2}$ test for percentages (independence in contingency tables); Kruskal-Wallis test for lengths of stay.

admission again, 204 of the 263 who survived to 180 days (78\%) would have wanted it. There was no significant difference in this respect between patients with COPD \pm and those in the "pure asthma" category.

\section{Predictors of quality of life on follow-up}

Patient-reported function in the follow-up questionnaire was slightly worse at 180 days than pre-admission function collected from either the patients themselves $(45 \%)$ or other witnesses (55\%) at admission to the ICU. Overall, about onehalf scored the same, about one-third were worse and about one-sixth were better. More detail on the changes in reported function for each pre-admission functional category is given in table 6. The actual agreement between function before admission to the ICU and on follow-up was quite low (kappa $=0.21, p<0.001$ ), but the correlation between the two (and therefore the discriminatory power of pre-admission function) was reasonably good (Spearman rank coefficient $=0.485, p<0.001$ ), so pre-admission functional status gives clinicians useful information about likely status on follow-up. As a rule of thumb, those who were housebound before admission will probably be the same at follow-up, but the most common (and equally likely) outcome for those who were fully mobile or independent is that they will stay the same or fall one category.

Doctors' predictions of quality of life at 180 days made on admission using the EuroQol VAS were slightly pessimistic compared with the patients' reported scores in the follow-up questionnaire (mean predicted score $50 \%$ vs $55 \%$ ). In spite of using 100 scale points rather than the 4 on the function scale, discrimination was relatively poor (Spearman rank coefficient $=0.221, p=0.0004)$. Agreement after converting both VAS scores into quartiles was also poor (kappa $=0.052$, $\mathrm{p}=0.032$.

\section{DISCUSSION}

\section{Limitations of study}

This study recruited patients with COPD, asthma or a mixture of asthma and COPD who were admitted to ICUs or HDUs in the UK with an episode of respiratory failure. The participating

Table 6 Function before admission and in the 180-day follow-up questionnaire

\begin{tabular}{llllll}
\hline \multirow{2}{*}{$\begin{array}{l}\text { Function in 2 weeks before } \\
\text { admission }\end{array}$} & \multicolumn{2}{l}{ Function reported in 180-day questionnaire } & & \\
\cline { 2 - 6 } & Fully mobile & Independent & Housebound & $\begin{array}{l}\text { Bed/ } \\
\text { chairbound }\end{array}$ & Total \\
\hline Fully mobile & 58 & 49 & 23 & 1 & 131 \\
& $44.3 \%$ & $37.4 \%$ & $17.6 \%$ & $0.8 \%$ & $100.0 \%$ \\
Independent & 26 & 65 & 67 & 2 & 160 \\
Housebound & $16.3 \%$ & $40.6 \%$ & $41.9 \%$ & $1.3 \%$ & $100.0 \%$ \\
Bed/chairbound & 7 & 26 & 74 & 13 & 120 \\
& $5.8 \%$ & $21.7 \%$ & $61.7 \%$ & $10.8 \%$ & $100.0 \%$ \\
Total & 0 & 1 & 7 & 1 & 9 \\
\hline
\end{tabular}


units and patients recruited appear to be representative of UK practice, and the rate of follow-up was good. In these respects the results should be generalisable and valid within the UK at least.

Importantly, however, all the patients in this study were selected for either ICU or HDU admission. Of the whole "population" of patients admitted to hospital with severe acute exacerbations of COPD, we would expect to have recruited relatively few on long-term oxygen and/or with low functional scores. (In one centre $48.9 \%$ of all hospitalised patients with COPD were housebound or worse compared with $38.2 \%$ in this study. ${ }^{12}$ ) Our overall results may therefore be least applicable to such patients. However, fig 1 suggests that the units taking part in the study had highly variable admission thresholds. The spectrum of those recruited - if not representative overall-was broad, with substantial numbers of patients with low functional scores, especially in the "not to be intubated" group. The results for subgroups are therefore of interest.

Assignment of the patients to diagnostic groups was based on clinical judgement at the time of admission. We cannot be certain that those classified as "pure asthma" really did have asthma or whether some of the younger patients, for example, had COPD. However, the 180-day survival rate for the COPD \pm group was approximately $60 \%$ compared with around $90 \%$ for the "pure asthma" group. Also, these diagnostic categories were significant predictors of prognosis in a multivariate prognostic model that took account of factors such as age and functional status. ${ }^{13}$

\section{Comparison with other studies}

On a scale of $0-100$, the mean rating by survivors of their overall quality of life was 54.9, significantly less than VAS scores for members of the public aged 65-74 years (mean 77.3) ${ }^{14}$ but similar to the mean (SD) score of 50.9 (16.4) for 132 outpatients with COPD of mean age 67 years and mean forced expiratory volume in $1 \mathrm{~s} 47 \%$ attending a chest clinic. ${ }^{15}$

Although they reported widespread discomfort and disability, around $75 \%$ of the responding survivors considered their quality of life to be similar to or better than it had been in the stable period before they were admitted, and over $95 \%$ of them would choose similar treatment again. This is broadly consistent with the results of an earlier study in which $87 \%$ of 81 patients with COPD of mean age 75 years said they would accept a "high burden" of treatment if it was life-saving and restored current health. ${ }^{16}$

In an earlier analysis we found that clinicians appeared to be pessimistic about the probability of survival of patients with COPD on admission to ICUs. ${ }^{4}$ This was also found in the SUPPORT study. ${ }^{2}$ Here we report that clinicians' predictions of the quality of life of survivors were undiscriminating as well as slightly pessimistic, but that functional status in the period of stability 2 weeks before ICU admission was a reasonable discriminator, if a slightly optimistic predictor, of function in the longer term. Again, these findings are consistent with results from other studies. Frick et $a l^{17}$ in the USA found that neither doctors nor nurses could predict outcomes well, and the SUPPORT study showed that functional status before ICU admission was a better indicator of longer-term function than doctors' predictions. ${ }^{18}$

This is the largest study to have systematically collected data on outcomes for patients with airway obstruction admitted to ICUs in the UK. Over 60\% survived for at least 180 days after ICU admission and, even among the relatively sick group who were "not to be intubated", 180-day survival was almost $50 \%$.
Functional capacity in the period of stability in the 2 weeks before hospital admission is a reasonably discriminating indicator of function at 180 days. Although survivors still had impaired quality of life, almost all of them would have wanted similar treatment again. We think that these findings should inform decision making for patients who are being considered for intubation.

Acknowledgements: The authors thank all the CMP units that participated in the study for recruiting the patients to make the study possible; the Intensive Care National Audit and Research Centre for their invaluable role in recruiting units and data linkage; and Jan Van Der Meulen for advice on the data analysis.

Funding: MW was funded by an MRC Health Services Research Fellowship. JG was funded by an NHS R \& D grant. The funder had no role in the design, analysis, interpretation or reporting of this research.

\section{Competing interests: None.}

Ethics approval: The study had multicentre research ethics committee approval.

Contributors: MJW, CS, KR, JA, BR and DY were involved in the original design of the study. MJW coordinated the study and carried out the initial data analysis and drafting of the paper. JG helped to collect the data. CS carried out additional analyses and drafted the final paper. KR and DH helped recruit the units and facilitated data linkage with the CMP. All the authors revised the manuscript critically for important intellectual content and approved the final version. MW is the guarantor.

\section{REFERENCES}

1. National Institute for Health and Clinical Excellence (NICE). Chronic obstructive pulmonary disease: national clinical guideline for management of chronic obstructive pulmonary disease in adults in primary and secondary care. Thorax 2004:59(Suppl I):152-4.

2. Connors A, Dawson N, Thomas C, et al. Outcomes following acute exacerbation of severe chronic obstructive lung disease. The SUPPORT investigators (Study to Understand Prognoses and Preferences for Outcomes and Risks of Treatments). Am J Respir Crit Care Med 1996;154:959-67.

3. Wildman M, O'Dea J, Kostopolou 0, et al. Variation in intubation decisions for patients with chronic obstructive pulmonary disease in one critical care network O J Med 2003;96:583-91

4. Wildman M, Sanderson C, Groves J, et al. Implications of prognostic pessimism in patients with chronic obstructive pulmonary disease (COPD) or asthma admitted to intensive care in the UK within the COPD and asthma outcome study (CAOS) multicentre observational cohort study. BMJ 2007:335:1132-5.

5. Harrison D, Brady A, Rowan K. Case mix, outcome and length of stay for admissions to adult, general critical care units in England, Wales and Northern Ireland: the Intensive Care National Audit \& Research Centre Case Mix Programme Database. Crit Care 2004;8:R99-111.

6. Menzies R, Gibbons W, Goldberg P. Determinants of weaning and survival among patients with COPD who require mechanical ventilation for acute respiratory failure. Chest 1989;95:398-405

7. Katz S, Ford A, Moskowitz R, et al. Studies of illness in the aged - the index of ADL: stndardized measure of biological and psychosocial function. JAMA 1963;185:914-9.

8. Brooks R, with the EuroOol Group. EuroQol: the current state of play. Health Policy 1996:37:53-72

9. Barley $\mathbf{E}$, Quirk F, Jones P. Asthma health status measurement in clinical practice: validity of a new short and simple instrument. Respir Med 1998:92:1207-14.

10. Wildman M, Harrison D, Welch C, et al. A new measure of acute physiological derangement for patients with exacerbations of obstructive airways disease: the COPD and Asthma Physiology score. Respir Med 2007:101:1994-2002.

11. Hill A. General principles of field surveys. In: The application of scientific methods to industrial and service medicine. London: Medical Research Council, 1951:16.

12. Wildman M, Groves J, Walia S, et al. Hospitalised COPD exacerbations: survival and univariate outcome predictors, 36 month follow-up. Thorax 2001:56(Suppl III):iii19.

13. Wildman $\mathbf{M}$, Sanderson C, Groves J, et al. Outcome prediction for admissions with COPD to UK critical care units from CAOS: the COPD and Asthma Outcome Study. Thorax 2004:59:S39.

14. Kind P, Dolan P, Gudex C, et al. Variations in population health status: results from a United Kingdom national questionnaire survey. BMJ 1998;316:736-41.

15. Harper R., Brazier J, Waterhouse J, et al. Comparison of outcome measures for patients with chronic obstructive pulmonary disease (COPD) in an outpatient setting Thorax 1997; 10:879-87.

16. Fried T, Bradley E, Towle M, et al. Understanding the treatment preferences of seriously ill patients. N Engl J Med 2002;346:1061-6.

17. Frick S, Uehlinger D, Zuercher Zenklusen R. Medical futility: predicting outcome of intensive care unit patients by nurses and doctors - a prospective comparative study. Crit Care Med 2003;31:456-61.

18. Wu A, Damiano A, Lynn J, et al. Predicting future functional status for seriously ill hospitalized adults. The SUPPORT prognostic model. Ann Intern Med 1995; 122:342-50 